\title{
Efforts sur une prise d'eau en mer comportant trois tours émergeantes. \\ Essais en bassin à houle aléatoire
}

\author{
Stresses on a water intake at sea \\ comprising three emerging towers
}

\author{
M. Canel \\ L. Barailler
}

Sogreah, Grenoble

L'étude d'une prise d'eau de centrale nucléaire en mer tyrrhénienne, comportant une plate-forme et trois tours, a été effectuée sur un modèle réduit en houle aléatoire déferlant sur les fonds marins. On a reproduit des spectres de Pierson-Moskowitz et Jonswap et mesuré les efforts globaux et les pressions sur la structure, ainsi que les efforts de liaison entre les tours et la plate-forme. L'appareillage de mesure comportait des sondes capacitives, une balance à six composantes et des dards pour l'encastrement des tours dans la plate-forme, avec acquisition digitalisée et traitement informatisé. L'étude a permis d'apprécier la stabilité globale de l'ouvrage et le coefficient de sécurité.

The study relating to the water intake, of a nuclear power station in the Tyrrhenian Sea, comprising a platform and three towers has been carried out on a scale model with random breaking waves. The Pierson-Moskowitz and Jonswap spectrums have been reproduced and the overall stresses and pressures on the structure, as well as the linking stresses between the towers and the platform, have been measured. The measurement equipment comprised capacitive sensors, a six component scale and darts for the embedding of towers in the platform, with digitalized acquisition and computerized processing. The study has made it possible to assess the overall stability of the works and the safety factor.

\section{Le problème et son contexte méthodologique}

Le dimensionnement des grandes structures gravitaires établies en mer peut avoir à répondre à trois types de préoccupations :

- La stabilité globale et les contraintes de fondation,

- la résistance des sections d'encastrement entre les différentes parties de la structure,

- la résistance des parois soumises aux pressions hydrodynamiques.

C'est précisément le cas de cet ouvrage de prise d'eau en mer que SOGREAH a eu récemment l'occasion d'étudier par des essais en laboratoire.
L'intérêt d'une approche expérimentale est surtout évident pour les deux premiers types d'objectifs fixés au dimensionnement. Elle autorise en effet la détermination globale, le modèle se chargeant d'assurer instantanément les plus complexes intégrations.

$\mathrm{Au}$ contraire, les méthodes alternatives par voie de calcul consistent généralement à déterminer les pressions hydrodynamiques élémentaires sur les parois pour un modèle de houle plus ou moins réaliste. Les nombreuses théories semi-empiriques que propose la littérature spécialisée ne conviennent que pour des formes simples et ne tiennent pas compte de l'altération de la houle par la structure étudiée. 

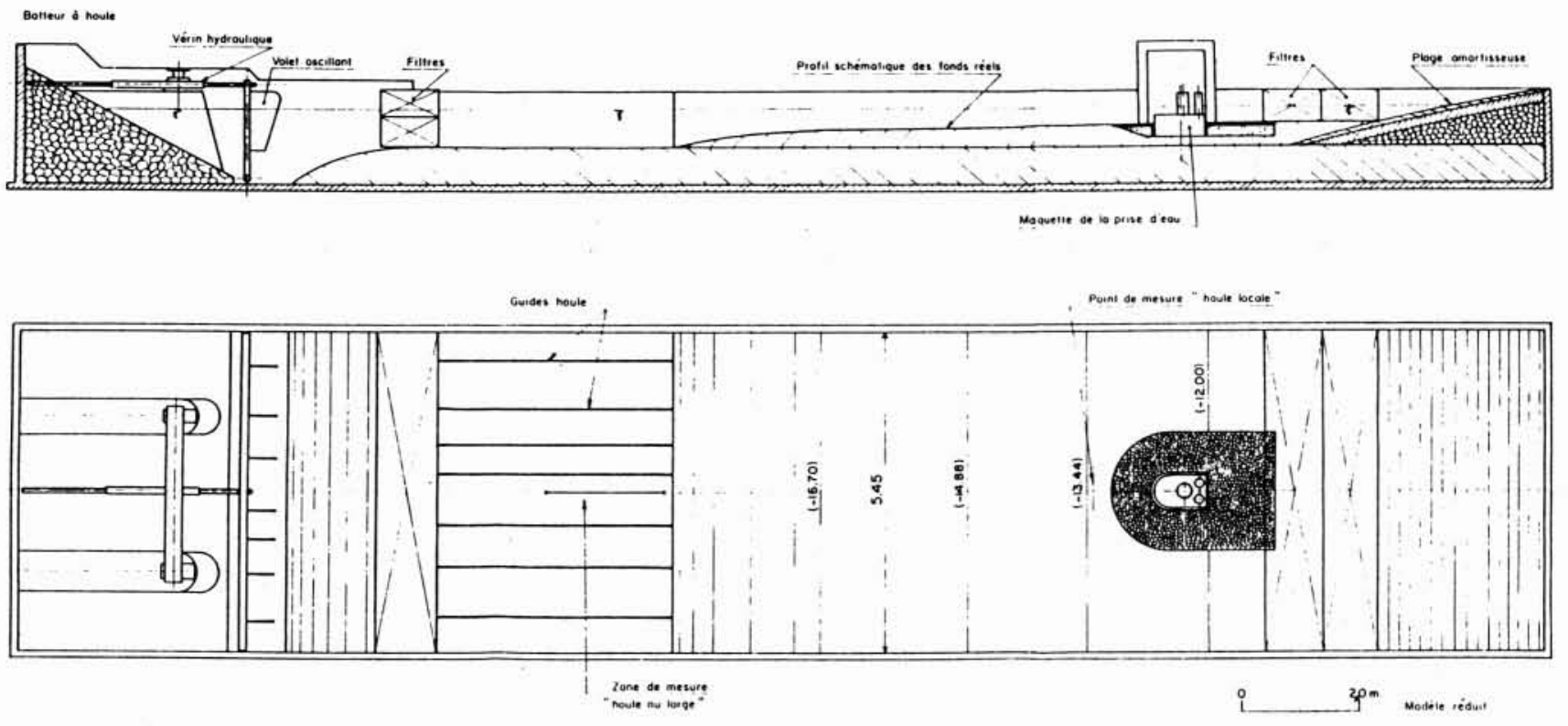

Figure 1. - Schéma de l'installation d'essai

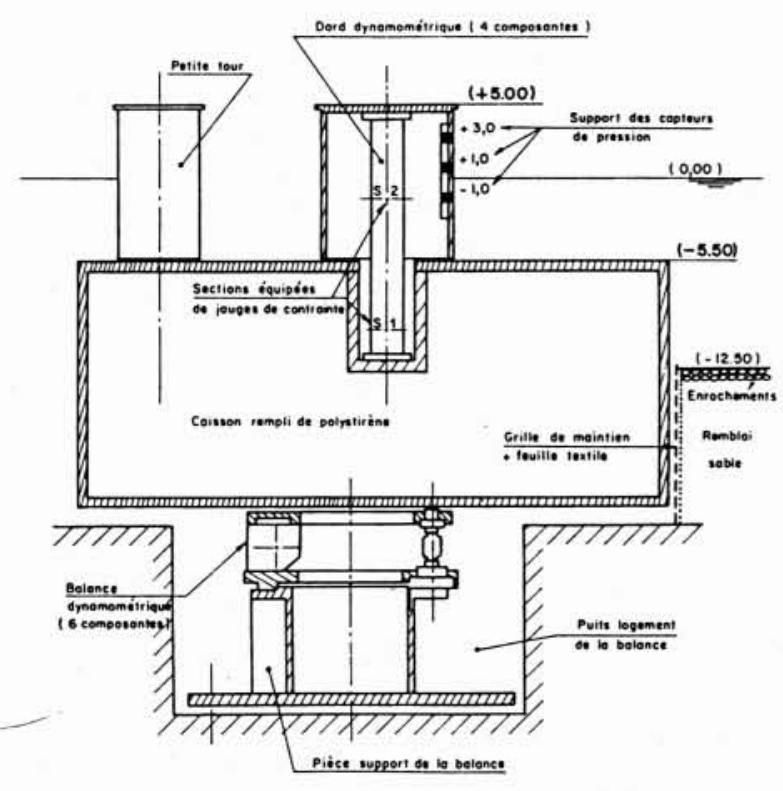

Figure 2. - Dispositif des mesures dynamométriques

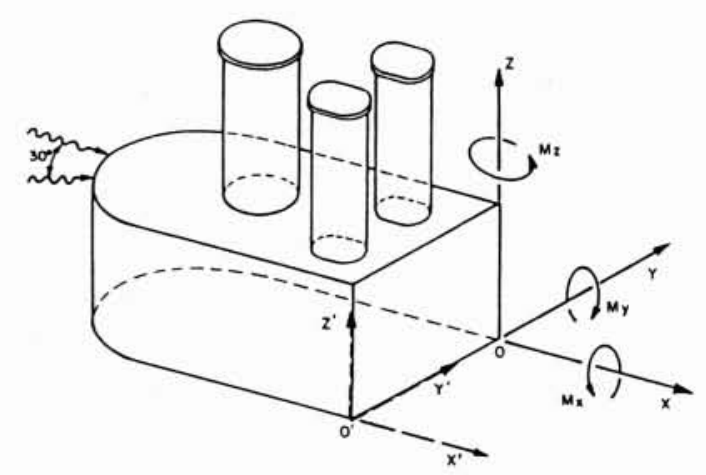

Figure 3. - Axes de référence des mesures dynamométriques 


\section{Le projet et son environnement}

Près du littoral d'un pays riverain de la Méditerranée occidentale, une centrale nucléaire est en cours de construction.

L'ouvrage de prise d'eau en mer sera une importante structure de béton armé $(39 \mathrm{~m} \times 24 \mathrm{~m} \times 16 \mathrm{~m})$ constituée d'un caisson submergé surmonté de trois grosses tours de visite qui émergent à la cote $(+5,0)$. L'ensemble doit être échoué par fonds de $(-12,5)$ dans une souille draguée à $(-22,0)$ puis remblayée au niveau du fond initial (cf. figures 1,2 et 3 ).

La morphologie des fonds du site est régulière et présente une pente constante de près de $2 \%$.

Le niveau moyen de la mer peut atteindre exceptionnellement la cote $(+1,00)$.

Les conditions de houle du projet sont définies sur la base d'une étude du régime des houles du large et majorées par des considérations relatives à la houle limite compte tenu de la bathymétrie :

- incidences extrêmes de 0 et $30^{\circ}$,

- types de distribution spectrale: Pierson-Moskowitz et Jonswap,

- périodes de pic : 12 et $14,3 \mathrm{~s}$,

- hauteur significative : de 7,5 à $8,0 \mathrm{~m}$,

- durée de tempête : 11,5 et 13,5 heures.

\section{Les conditions expérimentales}

Le caractère essentiellement gravitaire de la houle, l'importance des dimensions de la structure relativement aux longueurs d'onde de la houle et la quasi permanence du caractère turbulent de l'écoulement au contact de l'ouvrage (vagues déferlantes et arêtes vives du caisson) imposent pour de tels essais le choix de la similitude de Froude.

Bien que la représentation des effets de viscosité puissent ainsi être momentanément surestimés, ce risque de distorsion reste sans conséquence étant donné que, les principaux résultats retenus sont relatifs à des phases d'écoulement fortement turbulent.

Les dimensions du bassin et les performances du générateur de houle conduisent au choix de la plus grande échelle de réduction envisageable : $\lambda=1 / 43$

Le bassin à houle présente les dimensions principales suivantes (cf. figure 1 ) :

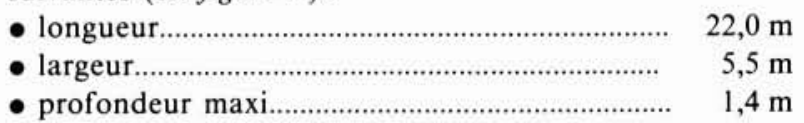

Le coefficient de réflexion du dispositif amortisseur est inférieur à $5 \%$.

Le générateur de houle permet la reproduction de la houle totalement aléatoire sur une conséquence de durée quelconque préalablement choisie.

Le batteur, dont le volet plan est articulé sur le fond, est actionné par un vérin hydraulique asservi. Un générateur électronique, synthétiseur de signaux aléatoires par filtrage de "bruit blanc", pilote ce vérin. Ce générateur permet la reproduction des distributions spectrales d'éner- gie classiques ou particulières et assure à la distribution des hauteurs de vagues incidentes une bonne conformité à la loi de Rayleigh $D^{\prime}$. L'évolution de cette dernière distribution caractéristique avec la durée de séquence aléatoire choisie est également correctement assurée.

La représentation bidimensionnelle des fonds d'implantation de l'ouvrage est réalisée dans le bassin par une chape de ciment troussé et lissé. La souille est également représentée et remblayée de sable; un filtre rigide maintient ce sable et évite tout contact avec la partie ensouillée de la maquette (cf. figure 2 ). La représentation d'incidences différentes est schématiquement réalisée par pivotement de l'ensemble des maquettes (souille-structure).

Réalisée en différentes pièces d'aluminium et de matière plastique rigide, cette maquette représente fidèlement toutes les formes extérieures de la prise d'eau. Pour des raisons d'instrumentation, les tours sont solidarisées au caisson par l'intermédiaire de dynamomètres très rigides.

L'instrumentation de mesure comporte (cf. figure 2) :

- un limnigraphe à sonde capacitive pour mesurer la houle;

- trois " dards " dynamométriques à jauges de contraintes sensibles en flexion pure, destinés à mesurer les deux composantes de l'effort tranchantjet du moment fléchissant dans la section d'encastrement de chaque tour sur le caisson:

- une "balance " dynamométrique à jauges de contrainte permettant la mesure des six composantes du torseur hydrodynamique global que la houle exerce sur l'ensemble de la maquette;

- six pressiomètres à jauges de contrainte montés sur supports-étuis amovibles qui permettent de mesurer la pression en différents points des parties exposées des tours.

Les signaux de ces capteurs sont adressés à un mini-ordinateur qui en assure la digitalisation - à raison de 25 valeurs par seconde - et l'enregistrement sur bande magnétique.

Des programmes de traitement numériques assurent en léger différé :

- les calculs matriciels de découplage des dynamomètres, - les analyses statistiques des écarts entre extremums successifs, des maxima et des minima conduisant à la détermination des histogrammes et des principales valeurs caractéristiques,

- les analyses spectrales suivant la méthode de Tukey.

Chaque essai comporte deux séquences identiques de deux heures de durée au cours de laquelle la houle est aléatoire. Au cours de chaque séquence 16 paramètres sont enregistrés simultanément.

\section{Les principaux résultats}

\subsection{Pressions locales}

Les valeurs maximales ne dépassent pas 3,2 bars pour la grosse tour et 2,5 bars pour la plus exposée des petites tours arrière. Malgré le caractère fortement déferlant des 
Exemples de diagrammes de fréquence de dépassement des valeurs maximales

DI - Houle mesurée devant la maquette (zone de déferlement).

D4 - Effort tranchant dans les sections d'encastrement des tours en caisson

D5 - $\mathrm{M}_{\mathrm{y}}$ : Moment de renversement longitudinal vers l'arrière

$\eta_{\mathrm{s}}$ : critère de glissement longitudinal.
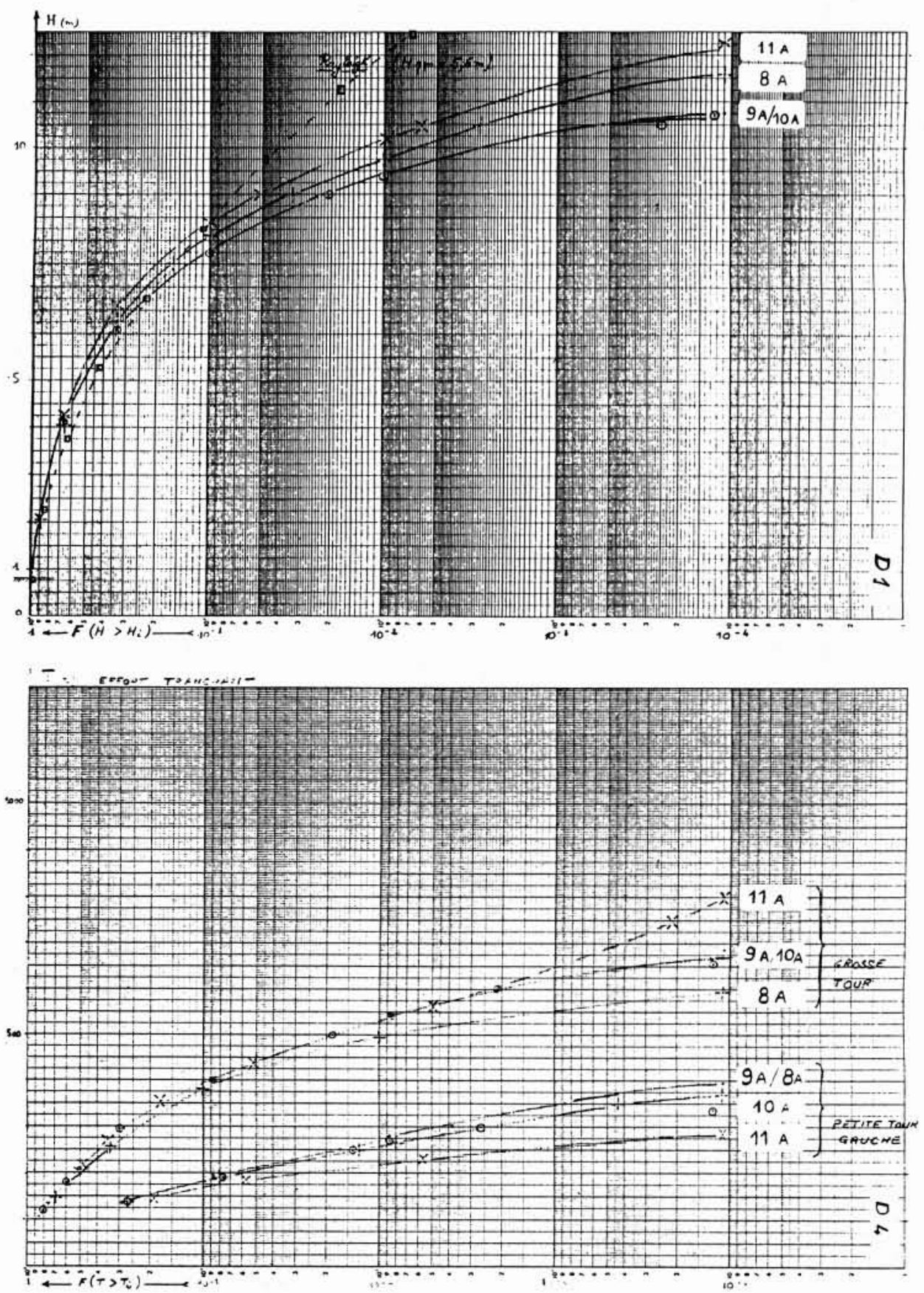
vagues, la forme des signaux paraît exclure le risque de " gifle".

La distribution instantanée des pressions sur une génératrice est sensiblement trapézoïdale, le maximum est toujours voisin de la surface libre. Le diagramme enveloppé de ces' distributions instantanées est une ligne polygonale dont le sommet, représentatif de la pression maximale enregistrée au cours d'une même tempête, se situe à proximité du niveau d'eau de repos.

\subsection{Efforts de liaison dans les sections d'encastrement des tours}

Les ordres de grandeur des valeurs maximales de l'effort tranchant et du moment fléchissant à la section de liaison de chaque tour avec le caisson sont respectivement :

- $800 \mathrm{t}$ et $4300 \mathrm{mt}$ pour la grosse tour, et en incidence nulle,

- $300 \mathrm{t}$ et $1700 \mathrm{mt}$ pour la petite tour gauche, que l'incidence soit nulle ou à $30^{\circ}$ du côté de cette tour,

- $400 \mathrm{t}$ et $2400 \mathrm{tm}$ pour la petite tour droite en incidence oblique à $30^{\circ}$ du côté opposé à cette tour.

En supposant simultanés les maxima du moment fléchissant et de l'effort tranchant, la cote du point d'application de la résultante horizontale resterait voisine de 0,00 à $+0,5$ et relativement indifférente à la cote du plan d'eau au repos, à l'incidence de la houle sur la structure et au type de spectre considéré.

\subsection{Stabilité globale (efforts hydrodynamiques glo- baux)}

L'effort maximum d'entraînement horizontal est de $2800 \mathrm{t}$ dans la direction de la houle et de $2000 \mathrm{t}$ dans la direction opposée.

L'effort de soulèvement maximum atteint près de $1700 \mathrm{t}$, l'effort maxi d'appui sur le fond dépasse légèrement $2000 \mathrm{t}$.

Les tours sont responsables de $50 \%$ du maxi de l'effort d'entraînement horizontal et de 25 à $30 \%$ des maxima d'effort vertical.

L'ouvrage étant considéré en configuration vide d'eau et orifices bâtardés, les vérifications sont faites sur la base de valeurs théoriques des paramètres de stabilité :

- poids $P=-2700 \mathrm{t} ; X_{\mathrm{g}}=-18,5 ; \quad Z_{\mathrm{R}}=+8,1$

- flottabilité $F=+15204 \mathrm{t} X_{c}=-18,0 ; \quad Z_{c}=+8,5$

Le critère de soulèvement, défini comme :

$$
\eta_{s}=\frac{F_{z}}{F+P}
$$

(avec $\eta,>-1$ comme condition de stabilité)

ne dépasse pas $-0,14$, soit un coefficient de sécurité supérieur à 7 .

En négligeant la résistance du remblai au glissement horizontal de la structure et en adoptant un coefficient de frottement de 0,5 sur la fondation, on définit le critère de stabilité au glissement par :

$$
\eta_{8}=\frac{2 F x y}{F_{z}+F+P}
$$

(avec $\eta_{8}>-1$ comme condition de stabilité)
Ce critère n'est pas inférieur à $-0,48$, valeur obtenue en houle normale.

L'examen de critères analogues pour caractériser les conditions de stabilité au renversement autour de l'arête arrière et des deux arêtes latérales du caisson montre que la sécurité est de 5,5 dans le pire des cas.

\section{Observations méthodologiques}

\subsection{A propos des pressions}

Sur les ouvrages réels, les mesures des pressions hydrodynamiques dans le cas de houles déferlantes sont toujours délicates et donnent des résultats très dispersés pour des raisons propres aux capteurs et la nature "mécanique " du fluide. Sur un modèle réduit, le risque supplémentaire de distorsion n'est pas à écarter, surtout si l'on a affaire à des impacts sous forme de "gifles " avec emprisonnement d'air. L'examen de la forme des signaux peut instruire sur le régime des fluctuations de pression et sur la similitude à considérer.

\subsection{A propos des efforts d'encastrement des tours}

Dans le projet considéré, ces mesures ont mis en évidence des différences de contraintes entre grosses et petites tours : la section de liaison de la grosse tour est 2,6 fois plus importante que celle d'une petite tour et n'est jamais sollicitée plus de deux fois que la plus sollicitée des petites tours.

D'autre part, il conviendrait d'analyser davantage les multiples aspects de la nature transitoire et alternée de ces modes de sollicitation du matériau, sans doute au plus grand profit des spécialistes des phénomènes de fatigue : par exemple, recherche de corrélation entre réponse spectrale et distribution des valeurs des dérivées premières et secondes des efforts par rapport au temps, des vitesses et accélérations de houle, des cambrures de vague, identification de risques de résonance.

\subsection{A propos de stabilité globale}

L'analyse effectuée dans le cadre de cette étude reste élémentaire. On a vu en effet que, pour rester simple et conservative, cette analyse ne tient pas compte de la participation des réactions mobilisables de butée et de frottement du matériau de remblai, mais qu'à l'inverse elle considère la fondation capable de "mobiliser" une véritable charnière sous les arêtes concernées par les moments de renversement de sens " ad hoc".

Il paraît d'autre part vraisemblable que le régime de ces sollicitations de basculement alternées à la fréquence des vagues doit conditionner le régime des pressions locales sur la plate-forme de fondation.

D'ailleurs, l'examen des phases relatives des moments longitudinal et transversal d'une part, et de l'effort vertical d'autre part, fait apparaître un synchronisme systématique au passage des maxima qui traduit de manière évidente une dynamique de poinçonnement notoire de l'angle arrière droit de la plate-forme de fondation; du même 
coup, cela suggère qu'il peut en résulter une certaine influence sur le mode de fatigue structurelle du fait de la répartition très contrastée des contraintes qu'elle préfigure.

\subsection{A propos de représentativité des valeurs maximales}

La pratique expérimentale en régime aléatoire conduit à caractériser les distributions statistiques des paramètrescauses ainsi que celles des paramètres-effets - données et résultats - par un certain nombre de valeurs représentatives de la fraction déterminante de ces différentes populations. De fait, la tendance pratique consiste le plus souvent à ne retenir que la valeur maximale de chaque paramètre.

Or, la valeur maximale seule n'est représentative que de l'essai particulier si la distribution de l'ensemble des valeurs est inconnue.

Dans le cas présent où la distribution des hauteurs des vagues incidentes au large est conforme au modèle de Rayleigh et où la durée est suffisante - près de 5000 vagues - pour conférer aux valeurs maximales une représentativité convenable, il est particulièrement intéressant d'examiner plus précisément les tendances que présentent les distributions des différents paramètres-effets dans le domaine des très faibles probabilités. Cette démarche est de nature à valoriser davantage la représentativité de ce résultat absolu par une meilleure connaissance du gradient de risque pour des probabilités un peu inférieures ou un peu supérieures.

Le premier examen de ce type, effectué sur la mesure de houle au large immédiat de la structure, met en évidence les conséquences du déferlement sur la déformation de la distribution des vagues fortes :

- diminution du nombre de vagues fortes au profit de celui des vagues moyennes et faibles,

- diminution du gradient de risque de voir dépassée la vague maximale.

En représentation semi-logarithmique, les fonctions de distribution des hauteurs de dépassement se voient modifiées d'une courbe parabolique :

$$
F\left(H>H_{i}\right)=e^{-(H / k)^{2}}
$$

en courbe de type exponentiel :

$$
F\left(H>H_{i}\right)=e^{-e^{H-h} a}
$$

qui rappelle la loi de Gumbel utilisée pour l'ajustement statistique à long terme des fréquences de dépassement de hauteur. Malgré l'importance de la durée de tempête considérée, le "plafonnement " total n'est pas atteint : à titre indicatif, dans le pire des cas, le risque de progression de $H$ est encore de $0,3 \mathrm{~m}-$ soit $2,5 \%$ du Hmax atteint - pour un doublement de la durée.

Le même type d'examen, pratiqué sur les résultats des mesures de pression sur les tours, révèle une relation de type presque linéaire entre la fréquence de dépassement et les valeurs des maxima de pression : la fonction de transfert (houle déferlante $\rightarrow$ pics de pression) ne serait pas linéaire. De plus, pour les valeurs du millième supérieur correspondant peut-être aux vagues fortement défer- lantes, une certaine dispersion affecte les valeurs de pic de pression qui ont tendance, dans certains cas, à augmenter plus rapidement avec la durée d'essai, c'est-à-dire avec les hauteurs des vagues déferlantes.

Les efforts tranchants dans les sections d'encastrement des tours au caisson suivent, à une exception près, des lois de fréquence de dépassement très analogues à celles des vagues déferlant devant la structure.

Les diagrammes de fréquence de dépassement relatifs aux sollicitations globales telles que $M_{\mathrm{s}}$ et $\eta_{\mathrm{s}}$ présentent également des allures analogues à celles des diagrammes relatifs à la houle devant la structure. A titre indicatif, le doublement de la durée de tempête aurait pour conséquence une majoration de :

- 3,5\% du maximum de $M_{1}$, d'où une diminution égale en terme de stabilité au renversement longitudinal en arrière,

- $4,5 \%$ du maximum de $\left|\eta_{k}\right|$ critère de glissement horizontal.

Ces différentes analyses, entreprises pour éclairer par l'examen des tendances des lois de fréquence de dépassement la représentativité des valeurs maximales, ont conduit à :

- la mise en évidence de la modification par le déferlement de la distribution des vagues incidentes;

- la conclusion que pour la plupart des paramètres mesurés par extensométrie globale, les valeurs maximales relatives à des tempêtes de 5000 vagues constituent des valeurs très représentatives qui ne risquent d'augmenter que de moins de $5 \%$ pour un doublement de la durée.

- la mise en évidence de certaines singularités en matière de statistique des pics de pression de rang élevé - ceux du millième supérieur - qui sembleraient devoir croître plus rapidement au-delà d'un seuil de hauteur déferlante.

\section{Discussion} Président : M. M. BANAL

M. Boulon: Vous avez parlé de pression dynamique de 3 bars 5 à 13 mètres de profondeur...

M. CANEL : Cette pression était mesurée sur les génératrices des tours, mais les pressions maximales se situaient au niveau de la surface de l'eau.

M. BOUlon: Cela fait des pressions absolument énormes dues aux vagues déferlantes ( 3,5 bar), ce qui impliquerait des vitesses non moins importantes, de l'ordre de $100 \mathrm{~km}$ /heure. Pour ma part, je ne suis pas spécialiste; est-ce une réalité maintes fois vérifiée ?

M. CANEL: Oui. 